\section{Early complete remission of osteoid osteoma with conservative medical management}

\author{
Masahiro Yokouchi, Satoshi Nagano, \\ Hirofumi Shimada, Shunsuke Nakamura, \\ Takao Setoguchi, Ichiro Kawamura, \\ Yasuhiro Ishidou, Setsuro Komiya \\ Department of Orthopedic Surgery, \\ Graduate School of Medical and Dental \\ Sciences, Kagoshima University, \\ Kagoshima, Japan
}

\begin{abstract}
Osteoid osteoma is a benign bone tumor and causes persistent pain that is usually treated by surgery or ablation therapy. Conservative management with non-steroidal anti-inflammatory drugs (NSAIDs) is also used to avoid the morbidity associated with surgery or ablation therapy; however, it usually takes several years for the condition to resolve using conservative treatment. Our patient, a 10 -year-old boy, presented with a 3-month history of a painful lesion in his leg. Plain radiography, bone scanning, computed tomography and magnetic resonance images showed the presence of a lesion with radiological features consistent with an osteoid osteoma of the cortex in the tibial diaphysis. The patient was treated with a usual dose of ibuprofen for 3 weeks. Within 3 weeks, his symptoms were almost completely resolved; he no longer needed NSAIDs and returned to normal life. Repeat imaging studies showed complete disappearance of the nidus within 2.5 years after the resolution of symptoms.
\end{abstract}

\section{Introduction}

Osteoid osteoma is a relatively common skeletal neoplasm that occurs in young people. The lesion is most commonly located in the cortex of long bones, especially in the proximal femur and tibia, and is smaller than $1.0 \mathrm{~cm} .^{1}$ The most common symptom of this tumor is severe aching pain that is usually worse at night. Although aspirin and non-steroidal antiinflammatory drugs (NSAIDs) can temporarily provide a dramatic pain relief, the unremitting nature of the patient's pain usually leads to a surgical referral.

Some authors successfully demonstrated and recommended prolonged NSAID therapy for the treatment of osteoid osteoma. ${ }^{2-4}$ It may be an effective treatment option without the morbidi- ties associated with surgery or ablation therapy. However, long-term administration of NSAIDs usually 2 years or more - is necessary to resolve the symptoms. A number of gastrointestinal complications have been reported due to prolonged treatment with NSAIDs.

In this report, we describe an exceedingly rare case of osteoid osteoma with early complete resolution of the symptoms after only 3 weeks of NSAID administration. Moreover, radiography showed that the radiolucent nidus completely regressed 2.5 years after symptom resolution. To the best of our knowledge, this case represents the quickest resolution of osteoid osteoma symptoms in a patient treated with conservative medical treatment.

\section{Case Report}

A previously healthy 10 -year-old boy visited a nearby hospital because of increasing pain and swelling in the area of his left leg that was first experienced a month ago. He had no history of previous trauma to the area of concern. His condition was diagnosed as osteomyelitis of the tibia and antibiotics were administrated. The patient showed no response to initial treatment for 2 months and was subsequently referred to our hospital. At his first visit to our hospital, he complained of severe nocturnal pain. Physical examination revealed mild swelling with tenderness in the middle part of his leg. Results of laboratory examinations indicated a normal white blood cell count, Creactive protein level, and erythrocyte sedimentation rate. Radiographs showed a lucent zone and a surrounding sclerotic zone in the middle third of the tibia (Figure 1A). Computed tomography (CT) revealed a $10-\mathrm{mm}$ circumscribed osteolytic area in the cortex of the tibia with surrounding sclerosis (Figure 1B). This radiographic appearance of a radiolucent nidus with surrounding sclerosis was consistent with the typical appearance of an osteoid osteoma. Moreover, a bone scan revealed the double-density sign, which is quite specific for diagnosis of osteoid osteoma (Figure 1C). ${ }^{1}$ Magnetic resonance (MR) images showed a 10-mm circumscribed abnormality located in the cortex of the tibia with surrounding soft tissue edema. The circumscribed signal abnormality was isointense in comparison with bone marrow on fat-suppressed T1-weighted images and was moderately enhanced by gadolinium (Figure 2A, B). Axial CT images of the lesion clearly demonstrated an osteolytic lesion corresponding to the abnormality seen on MR images. The patient's symptoms markedly improved with NSAIDs and his parents did not provide consent for surgical treatment. He was therefore treated with a usual dose of ibuprofen (300
Correspondence: Masahiro Yokouchi, Department of Orthopedic Surgery, Graduate School of Medical and Dental Sciences, Kagoshima University, 8-35-1 Sakuragaoka, Kagoshima, 890-8520, Japan.

Tel. +81.99.275.5381 - Fax: +81.99.265.4699.

E-mail: masahiro@m3.kufm.kagoshima-u.ac.jp

Key words: osteoid osteoma, conservative therapy, NSAIDs, radiological resolution.

Contributions: MY managed the case and drafted the manuscript. SN, HS, NS, TS, IK, and YI collected the findings and participated in the design of the study. SK was involved in the design of the study and study coordination and helped draft the manuscript.

Conflict of interests: the authors declare no potential conflict of interests.

Received for publication: 16 January 2014.

Revision received: 6 February 2014

Accepted for publication: 6 February 2014.

This work is licensed under a Creative Commons Attribution NonCommercial 3.0 License (CC BYNC 3.0).

(C) Copyright M. Yokouchi et al., 2014

Licensee PAGEPress, Italy

Pediatric Reports 2014; 6:5311

doi:10.4081/pr.2014.5311

$\mathrm{mg} /$ day, 3 times daily) for 3 weeks. Within 3 weeks, his symptoms were almost completely resolved; he no longer needed NSAIDs and returned to normal life. Serial images including radiographs, CT scans and MR images revealed complete radiological resolution of the nidus 2.5 years after initial presentation (Figure 3). An early complete remission of osteoid osteoma with conservative medical treatment was confirmed in this patient.

At the 5-year follow-up visit, patient was free of pain and there was no evidence of recurrence.

\section{Discussion}

Osteoid osteoma most commonly arises in the cortex of long bones. Its radiographic features consist of a central oval or round nidus surrounded first by a radiolucent area followed by another area of sclerotic bone. The radiographic features are usually distinctive, ${ }^{5-7}$ and the clinical picture is characteristic. Therefore a diagnosis of osteoid osteoma is often made based on typical radiographic and clinical features. ${ }^{8}$ On the other hand, Sim et al. ${ }^{9}$ described that other conditions can manifest as osteoid osteoma on imaging that should be considerd such as Brodie abscess. In our case, the diagnosis of this lesion as an osteoid osteoma is 
based on radiographic and clinical data without histological proof. The possibility this may actually represent some other lesion such as Brodie abscess or an atypical stress fracture can not be excluded. ${ }^{9}$

There is a general agreement in the literature that complete removal of the nidus is the treatment of choice and that incomplete removal of the nidus leads to an increased rate of symptom recurrence. ${ }^{10}$ Therefore, surgical removal of the nidus has long been considered the gold standard in the treatment of osteoid osteoma. However, this procedure is not without risks. Accurate intraoperative assessment of the localization of the tumor edges is difficult and often results in unnecessary wide bone resection to ensure adequate margins. As a result, patients often need internal fixation and have a prolonged recovery period.1,11

Recent minimally invasive techniques such as CT-guided core-drill excision and radiofrequency or laser ablation have emerged as alternative methods for conventional surgical excision. In fact, these procedures are attractive treatment options and are considered not only as safe and effective, but also as the treatment of choice for osteoid osteoma.1,12,13 In particular, these methods have advantages over conventional surgery in cases of deep lesions such as those in the neck of the femur. However, these procedures are still associated with risks and are invasive treatment options. ${ }^{12,13}$

Conservative medical management with NSAIDs is also reported as an important option without the morbidities associated with surgery or ablation therapy. ${ }^{2-4,14,15}$ Increased local concentration of prostaglandins have been found to be produced within the nidus, explaining the excellent response to NSAIDs. ${ }^{1}$ The general mechanism of action of NSAIDs is inhibition of the synthesis of prostaglandins, so the treatment is specific. Kneisl and Simon demonstrated that conservative medical treatment with NSAIDs in 9 patients with osteoid osteoma resulted in long-term outcomes similar to those with surgical treatment. ${ }^{3}$ However they suggested that long-term administration of NSAIDs (mean treatment period of 33 months) is necessary before discontinuation of NSAID treatment for sustained symptom resolution. In their study, 3 of 9 patients (33\%) did not experience complete symptom relief without the use of NSAIDs. Goto et al. reported similar outcomes in 12 patients treated with NSAIDs. They demonstrated that all patients maintained a pain-free state; however, mean treatment period with NSAIDs in 8 patients was 18.3 month (range, 2-36 months). 15 Several other reports also demonstrated that conservative management with NSAIDs takes years before the condition is resolved.2-4 It is well known that the long-term use of NSAIDs is associated with a high incidence of serious side effects including a number of gastroin-

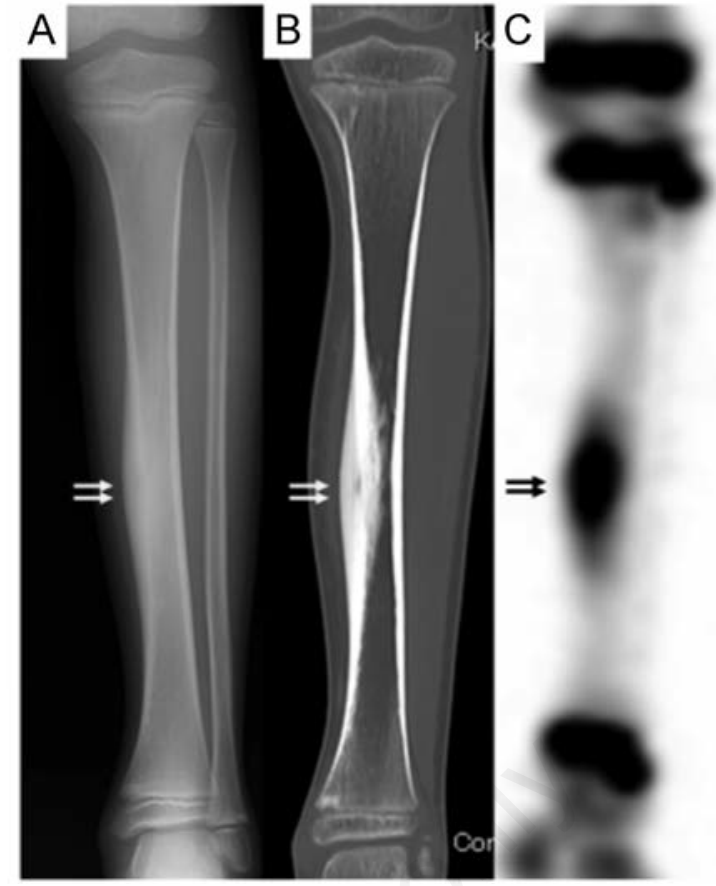

Figure 1. A) Anteroposterior radiographs showing a lucent zone and a surrounding sclerotic zone in the middle third of the tibia. B) Direct coronal computed tomography scan showing a 10-mm circumscribed osteolytic area in the cortex of the tibia with surrounding sclerosis. C) A bone scan showing an area of intense focal uptake with a surrounding area of lesser uptake, which is characteristic scintigraphic pattern of osteoid osteoma.

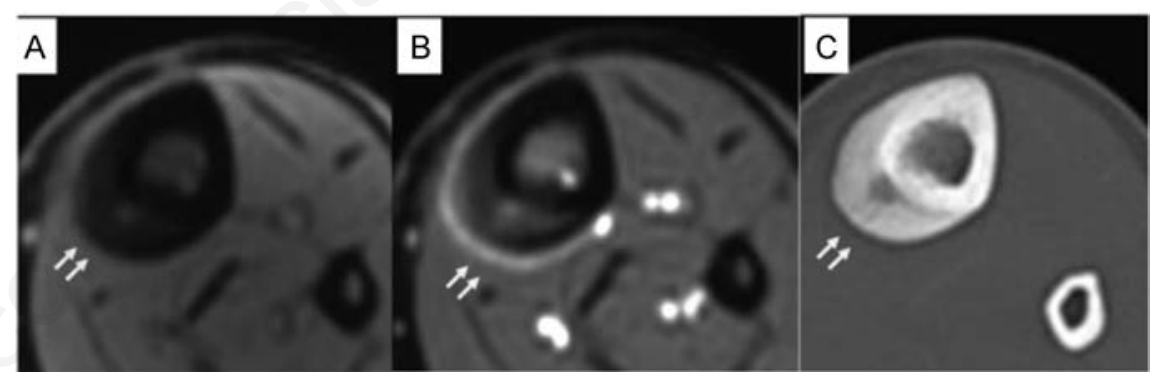

Figure 2. A) Fat-suppressed T1-weighted magnetic resonance image showing a 10-mm circumscribed abnormality located in the cortex of the tibia. B) Gadolinium enhanced fat-suppressed T1-weighted image showing a circumscribed signal abnormality in the cortex of the tibia that was moderately enhanced. C) Axial computed tomography image of the patient clearly showing an osteolytic lesion corresponding to the abnormality seen on magnetic resonance imaging.

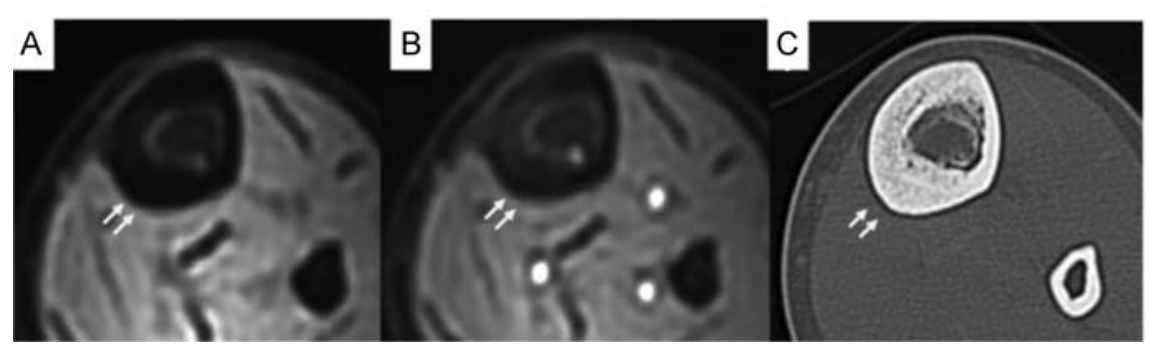

Figure 3. Repeat computed tomography and magnetic resonance imaging indicate radiological resolution of the nidus 2.5 years after initial presentation. A) Fat-suppressed T1weighted magnetic resonance image: 30 months after initial presentation. B) Gadolinium enhanced fat-suppressed T1-weighted magnetic resonance image: 30 months after initial presentation. C) Axial computed tomography image: 30 months after initial presentation. 
testinal complications. Hence, because of the chronic use of NSAIDs and the increased risk of gastrointestinal complications, conservative medical management is not considered the gold standard in managing osteoid osteoma; rather, it is as an option for those unwilling to undergo an invasive procedure.

In this report, we describe the case of a patient with osteoid osteoma who showed early complete symptom resolution with only 3 weeks of NSAID administration. The risk of gastrointestinal complications associated with short-term administration of NSAIDs is much lower than associated with long-term administration. In addition, recently developed cyclooxygenase- 2 selective inhibitors as well as proton inhibitors have decreased the risk of gastrointestinal toxicity in the treatment of osteoid osteoma. ${ }^{15}$ Although our case was extraordinarily rare, several reports of patients who are successfully treated with short-term administration of NSAIDs (2 to 3 months) exist in the literature. 15 Therefore, we believe that patients with osteoid osteoma should be provided information on early complete remission of pain with medical management.

Repeat imaging studies in our patient showed sclerosis and thickening of the cortex persisted, in spite of the early complete disappearance of the nidus. In previous study, regression of the nidus with persistent cortical thickening in conservatively managed patients also demonstrated.16,17 However, the reason why sclerotic change took so long to remodel is so far unclear.

This case report illustrates how osteoid osteoma may sometimes respond quite quickly to anti-inflammatory agents suggesting that a trial of NSAIDS such be considered in patients with osteoid osteoma.

\section{Conclusions}

We present a rare case of clinically diagnosed osteoid osteoma in which early complete resolution of the symptoms as well as the nidus was noted with conservative medical management.

\section{References}

1. Boscainos PJ, Cousins GR, Kulshreshtha $\mathrm{R}$, et al. Osteoid osteoma. Orthopedics 2013;36:792-800.

2. Leicester AW, Trantalis JN. Osteoid osteoma in a young child: successful non-operative management. ANZ J Surg 2001;71: 491-3.

3. Kneisl JS, Simon MA. Medical management compared with operative treatment for osteoid-osteoma. J Bone Joint Surg Am 1992;74:179-85.

4. Bottner F, Roedl R, Wortler K, et al. Cyclooxygenase-2 inhibitor for pain management in osteoid osteoma. Clin Orthop Relat Res 2001;393:258-63.

5. Brailsford JF. The radiology of bone and joints. Ed.5. Baltimore: The Williams and Wilkins Co; 1953.

6. Edeiken J, Hodes PJ. Roentgen diagnosis of bone. Baltimore: The Williams and Wilkins Co; 1967.

7. Freiberger RH, Loitman BS, Helpern M, Thompson TC. Osteoid osteoma; a report on 80 cases. Am J Roentgenol Radium Ther Nucl Med 1959;194-205.

8. Simm RJ. The natural history of osteoid osteoma. Aust NZ J Surg 1975;45:412-5.

9. Sim FH, Dahlin CD, Beabout JW. Osteoidosteoma: diagnostic problems. J Bone Joint Surg Am 1975;57:154-9.

10. Regan MW, Galey JP, Oakeshott RD. Recurrent osteoid osteoma. Case report with a ten-year asymptomatic interval. Clin Orthop Relat Res 1990;253:221-4.

11. Rosenthal DI, Hornicek FJ, Wolfe MW, et al. Decreasing length of hospital stay in treatment of osteoid osteoma. Clin Orthop 1999;361:186-91.

12. Lanza E, Thouvenin Y, Viala $\mathrm{P}$, et al. Osteoid osteoma treated by percutaneous thermal ablation: when do we fail? A systematic review and guidelines for future reporting. Cardiovasc Intervent Radiol 2013. [Epub ahead of print]

13. Rosenthal DI, Hornicek FJ, Torriani M, et al. Osteoid osteoma: percutaneous treatment with radiofrequency energy. Radiology 2003;229:171-5.

14. Jayakumar P, Harish S, Nnadi C, et al. Symptomatic resolution of spinal osteoid osteoma with conservative management: imaging correlation. Skeletal Radiol 2007; $36: S 72-6$.

15. Goto T, Shinoda Y, Okuma T, et al. Administration of nonsteroidal antiinflammatory drugs accelerates spontaneous healing of osteoid osteoma. Arch Orthop Trauma Surg 2011;131:619-25.

16. Yanagawa T, Watanabe H, Shinozaki T, et al. The natural history of disappearing bone tumours and tumour-like conditions. Clin Radiol 2001;56:877-86.

17. Spouge AR, Thain LM. Osteoid osteoma: MR imaging of two untreated lesions. Clin Imaging 1999;23:254-8. 